\section{SEXO Y MODERNIDAD EN LA ESPAÑA DE LA SEGUNDA REPÚBLICA. LOS DISCURSOS DE LA CIENCIA}

\author{
Rafael Huertas* y Enric Novella** \\ *Instituto de Historia, CCHS, CSIC \\ rafael.huertas@cchs.csic.es \\ **Université du Luxembourg \\ enric.novella@uni.lu
}

Cómo citar este artículo/Citation: Huertas, R. y Novella, E. (2013). "Sexo y Modernidad en la España de la Segunda República. Los discursos de la ciencia". Arbor, 189 (764). a090. doi: http://dx.doi.org/10.3989/arbor.2013.764n6013

Recibido: 3 junio 2013. Aceptado: 18 septiembre 2013.

RESUMEN: Este artículo pretende estudiar los contenidos científicos del movimiento de "reforma sexual" que tuvo lugar en España en los años veinte y treinta y que culminó durante la Segunda República. Se abordan, al respecto, tres aspectos fundamentales: 1) la introducción de la nueva ciencia sexológica; 2) el interés por la educación sexual de los niños; y 3) la promulgación de una nueva legislación matrimonial (la ley de divorcio de 1932). Se identifican discursos científicos, asimilados a una "modernización" de las costumbres, siempre desde presupuestos laicos y progresistas. Se apunta la confrontación entre enfoques biologicistas o endocrinológicos y planteamientos psicoanalíticos, estableciéndose como eje argumental el fomento de una vivencia consciente y sana de la sexualidad. Temas como paternidad responsable, control de la natalidad, prevención de los trastornos mentales (ligados a vivencias sexuales traumáticas), etc., son analizados a la luz de fuentes médicas, psicológicas y jurídicas en relación con la reforma de las costumbres sexuales y la superación de la doble moral burguesa.

PALABRAS CLAVE: Segunda República; Sexología; Educación sexual; Psiquiatría; Psicoanálisis.

\section{SEX AND MODERNITY IN THE SECOND SPANISH REPUBLIC. THE SCIENCE DISCOURSES}

Copyright: (C) 2013 CSIC. Este es un artículo de acceso abierto distribuido bajo los términos de la licencia Creative Commons Attribution-Non Commercial (by-nc) Spain 3.0.

ABSTRACT: The aim of this article is to explore the scientific content of the "sexual reform" movement which took place in Spain during the 1920s and 1930s, culminating during the Second Republic. It covers three essential aspects of this subject: 1) the introduction of the new science of sexology; 2) the interest in sex education for children; and 3) the passing of new marriage legislation (the Divorce Law of 1932). Areas of scientific discourse are identified, associated with a "modernization" of mores and based on secular and progressive assumptions. The conflict between biological or endocrinological approaches and psychoanalytical methods is discussed, with encouragement of a healthy, aware experience of sexuality becoming established as the central argument. Issues such as responsible parenting, birth control, prevention of mental disorders (linked to traumatic sexual experiences), etc. are analysed in the light of medical, psychological and legal sources, in relation to the reform of sexual mores and overcoming the bourgeois moral double standard.

KEYWORDS: Second Spanish Republic; Sexology; Sex education; Psychiatry; Psychoanalysis. 


\section{INTRODUCCIÓN}

Durante las primeras décadas del siglo XX se produjeron en España una larga serie de iniciativas de divulgación científica y de actuación social y política que tenían como objetivo una profunda renovación de las ideas y los conceptos en torno a la sexualidad. Médicos como Marañón, Juarros, Lafora, Sanchis Banús o Garma, juristas como Jiménez de Asúa o ensayistas como Hildegart participaron de manera muy activa en este proceso a través de conferencias y publicaciones, pero también con la creación de instituciones o asociaciones como el Instituto de Medicina Social de Madrid o la Liga Española para la Reforma Sexual sobre Bases Científicas (1932) - sección española de la Liga Mundial para la Reforma Sexual- o con la promoción y el aval de importantes reformas legislativas como la Ley de Divorcio de 1932.

La España de principios del siglo XX se caracterizaba por unas condiciones económicas, una estructura política y una situación social muy controladas por una oligarquía dirigente fuertemente arropada por la Iglesia católica. La moral católica, de la que la represión sexual era, sin duda, uno de sus más destacados objetivos, ejercía una gran influencia cultural sobre la población, contribuyendo a la subjetivación o interiorización de las normas y el orden burgués (Vázquez y Moreno Mengibar, 1997; Seoane, 2010). En este contexto político y social se fueron desplegando, poco a poco, una serie de discursos en torno a la sexualidad muy diferentes a los del pensamiento hegemónico. En líneas generales, puede decirse que aquellos años asistieron a un enfrentamiento dialéctico entre las posturas más conservadoras, defensoras de la moral católica y la norma burguesa, y las más progresistas, promotoras de una "modernidad" sexual inseparable del necesario cambio social. Por un lado, las "fuerzas anti-sistema" de inspiración anarquista fueron las que con más amplitud acometieron la lucha por la liberación sexual como un capítulo insoslayable de la lucha contra la moral burguesa y por la revolución (Cleminson, 1995; Nash, 1995). Por el otro, una importante elite profesional de médicos y psiquiatras argumentaron científicamente la necesidad de reformar las costumbres sexuales, insistiendo en la necesidad de controlar la natalidad (Álvarez, 1990) y abogando, sobre todo, por la educación sexual como medio para garantizar una paternidad responsable, combatir problemas sanitarios importantes - como la mortalidad infantil y materna, las enfermedades venéreas, etc.y prevenir determinados trastornos mentales supuestamente relacionados con la represión sexual. En ocasiones, el ideario libertario y el discurso científico coincidieron, como en el caso del médico anarquista Félix Martí Ibáñez, responsable de la sección titulada "Consultorio Psíquico Sexual", publicada en la revista anarquista Estudios durante los años 1936 y 37 (Martí Ibáñez, 1976).

En las páginas que siguen pretendemos analizar los contenidos científicos de todo este movimiento por la "modernidad" y la libertad sexual en España a propósito de tres aspectos fundamentales: la introducción de la nueva ciencia sexológica, el interés por la educación sexual de los niños y la promulgación de una nueva legislación matrimonial. Estos discursos laicos y progresistas, en los que convivieron y se confrontaron enfoques biologicistas o endocrinológicos y planteamientos psicoanalíticos, tenían como eje argumental el fomento de una vivencia "ilustrada", "consciente" y "sana" de la sexualidad y, por tanto, la prevención de los trastornos mentales tanto en la infancia como en la edad adulta. Resulta especialmente significativo que todo este movimiento tuviera como telón de fondo un Estado en crisis, pero también una sociedad en amplia transformación y sometida a un complejo y en ocasiones contradictorio proceso de modernización económica, política, cultural y urbanística que se truncó con la dictadura franquista tras la Guerra Civil que asoló el país.

\section{CIENCIA Y REFORMA SEXUAL}

Aunque existe en España una cierta literatura sexológica publicada en el siglo XIX (Sánchez Granjel, 1974), es a comienzos del XX cuando se detecta un aumento cada vez más importante de la misma. Durante los primeros años del siglo no solo se publicaron las traducciones de obras extranjeras importantes - como las de Havelock Ellis (entre 1906 y 1913) o Auguste Forel (en 1912) - sino también las primeras monografías y ensayos de educación sexual debidos a autores españoles, entre los que destacan, ya en la segunda década del siglo, los de Antonio Piga (1910) y César Juarros (s.a.), ambos vinculados al Instituto de Medicina Social de Madrid (Álvarez, 1988).

Pero no fue hasta los años veinte - durante la Dictadura de Primo de Rivera y debido en parte al desencanto de los intelectuales y los profesionales hacia la misma - cuando surgieron las primeras iniciativas reformadoras. La "reforma sexual" fue propuesta y defendida entonces desde medios de expresión como la prensa diaria o diversas publicaciones que abordaban diferentes aspectos de la sexualidad (Álvarez, 2004), entre las que cabe destacar amplias coleccio- 
nes populares de divulgación sexual como la Pequeña Enciclopedia de Educación Sexual (Sevilla, 1932), la Biblioteca de Educación Sexual (Barcelona, 1932-33), Cultura Sexual (Barcelona, 1936-37) y, sobre todo, Temas Sexuales (Madrid, 1932-34), dirigida, esta última, por Ángel Martín Lucenay (Cleminson, 2004). Esta amplitud de oferta permite que nos hagamos una idea del interés por la sexualidad presente en la España del momento (Guereña, 2013, 69 y ss.), pero también de la importancia cultural de la "literatura popular" en los nuevos hábitos de lectura que la población urbana fue adquiriendo durante los años de la República (Santonja, 1989). Además de esta producción escrita, es necesario recordar conferencias y debates en ateneos y otras organizaciones como la Sociedad de Abolicionismo de la Prostitución - presidida por Juarros desde 1922 y que pretendía, además de una reforma de la reglamentación de la prostitución, una amplia campaña de higiene y educación sexual-, la Liga de Educación Social - fundada en 1928 y de orientación netamente feminista - o la Sociedad de Amigos del Niño - que promovería la celebración de un Primer Curso Eugénico Español que fue finalmente suspendido por el gobierno de la dictadura por entender que propiciaba el "regodeo pornográfico" (Barrachina, 2004)-.

Esta actividad reformista fue continuada, ya sin tantas cortapisas gubernamentales, durante los años de la República. Así, en 1932, se creó la Liga Española para la Reforma Sexual sobre Bases Científicas - filial de la "Weltliga für Sexualreform" fundada en 1928 en Berlín por Magnus Hirschfeld- que tuvo como órgano de expresión la revista Sexus y fue capaz de organizar las Primeras Jornadas Eugénicas Españolas, retomando el talante de aquel Primer Curso Eugénico que, años antes, no había podido celebrarse (Noguera, 1934). La Liga estuvo apoyada desde sus inicios por influyentes personalidades de la ciencia y de la cultura española como Gregorio Marañón, que fue su primer presidente, y Hildegart Rodríguez, una de las más importantes publicistas de la "liberación sexual", que fue su secretaria de organización (Sinclair, 2003).

Marañón era, sin discusión, uno de los médicos más prestigiosos del momento (Balaguer, 2013). En consonancia con su visión biológica y determinista de la sexualidad (Castejón, 2013), definió las categorías de masculinidad, feminidad y los estados intersexuales desde un punto de vista eminentemente endocrinológico, lo que le llevó a aceptar la superioridad física, intelectual y social del hombre y a insistir en que la función de la mujer debía ser la maternidad (Marañón, 1915). En relación a la homosexualidad y a las demás "perversiones", las consideraba enfermedades debidas a factores hormonales alterados (Marañón, 1929). No entraremos aquí en un análisis detallado de la problemática de las sexualidades marginales; baste decir que los años veinte y treinta fueron un periodo de visibilidad e identificación de la homosexualidad en España que ha sido objeto de interesantes trabajos en los últimos años (Álvarez, 2004; Cleminson, 2004; Vázquez y Cleminson, 2011). Pero, frente al determinismo y al biologicismo de Marañón, merece la pena detenerse brevemente en la figura y la obra de Hildegart Rodríguez, porque representa muy bien el talante más progresista de este discurso de reforma sexual sobre bases científicas, por más que su trágica y peculiar historia personal haya tendido a difuminar su importancia como gran adalid de la modernidad sexual en la España de la Segunda República ${ }^{1}$.

Toda la producción escrita de Hildegart, independientemente de que se tratase de folletos de divulgación o de obras con pretensiones más científicas, tuvo un hilo conductor común y un objetivo esencial: la libertad sexual. En este sentido, su discurso era fundamentalmente ideológico pero, para poder llevarlo a cabo, necesitaba apoyarse en argumentos científicos. Tal como ella misma decía, "la revolución científica conmueve no sólo este plano sexual y moral, sino los planos jurídicos y políticos de la sociedad" (Hildegart, [1931a] 1971, 37). La mayor parte de sus publicaciones aparecieron en forma de artículos periodísticos o folletos de divulgación como Educación sexual (1931b), pero también publicó extensas monografías, entre las que cabe citar La rebeldía sexual de la juventud (1931c) - publicada un año antes que Der sexuelle Kampf der Jugend [La lucha sexual de los jóvenes] (1932) de Wilhelm Reich-o Malthusismo y Neomalthusismo. El control de la natalidad (1932), que constituye una aportación relevante y en la que la autora demuestra un conocimiento amplio y actualizado del tema que aborda.

En cuanto a las relaciones de pareja, Hildegart hace unos planteamientos sumamente avanzados para la época. De hecho, su defensa de la libertad sexual llega a considerar la posibilidad "natural" tanto de la heterosexualidad como de la homosexualidad, y tanto de la monogamia como de la poligamia. Sin embargo, critica duramente la prostitución, por entender que no solo degrada a la mujer, sino que es uno de los baluartes fundamentales de la "doble moral burguesa". Para Hildegart, sólo la libertad sexual permitiría superar este tipo de situaciones: 
"La disminución de la prostitución (el heterismo) se llevaría a cabo si las mujeres fueran totalmente libres en el amor, actuando donde quisieran y con quien quisieran, pues solo entonces aminoraría el secreto afán de buscar en la prostituta una satisfacción que su capacidad económica o moral le veda hallar en el hogar tradicional" (Hildegart, [1931] 1971, 46).

Otro de los elementos fundamentales en la obra de Hildegart es la importancia que otorga a la anticoncepción. Dedica así algunos escritos a la "paternidad voluntaria" en los que describe "científicamente" los diversos métodos anticonceptivos que deben ponerse a disposición del proletariado para evitar la "epidemia terrible de las familias numerosas". Para Hildegart, la maternidad no deseada "oprime" la libertad de la mujer, aunque también afecta al hombre. En su opinión, la posibilidad de mantener relaciones sexuales sin el temor a un embarazo no deseado resulta fundamental en el desarrollo de una sexualidad más libre, y ello debe traducirse tanto en la elección del goce sexual como en la de la maternidad: "La libertad habrá de vivir siempre en guardia, porque será [...] la generadora de la paternidad consciente, siempre que se desee y se esté en condiciones físicas de soportarla" (Hildegart, 1931, 66).

Pero el pensamiento reformista de Hildegart no se conforma con la trasformación de la moral y las costumbres sexuales, reclamando del Estado una participación decidida en la creación de instituciones para el cuidado de los hijos, otorgando una gran importancia al seguro de maternidad e insistiendo en que las mujeres debían acogerse a dicho seguro sin reticencias. Como es sabido, el seguro de maternidad fue un elemento destacado del paquete de políticas sociales puestas en marcha durante el primer bienio republicano, aunque existieron resistencias populares por parte de las mujeres trabajadoras, bien por no querer pagar las cuotas, bien porque, siendo solteras, lo consideraban inútil o deshonroso (Rodríguez Ocaña, 1990, 336).

Es evidente, pues, que Hildegart representa una de las vertientes más progresistas, críticas y emancipatorias del pensamiento español de su época. Su juventud y la frescura y audacia de sus argumentos deslumbraron a intelectuales, científicos y políticos, hasta el punto de que llegó a cartearse con reputados especialistas internacionales como el propio Havelock Ellis. El respeto intelectual y el interés que el trabajo de Hildegart suscitó en este médico inglés, partidario del socialismo fabiano, queda de manifiesto en un breve texto titulado "La virgen roja", en el que hace referencia a "la mente finamente equilibrada de Hildegart" (Ellis, 1933, 175) y elogia sus aportaciones a la sexología.

En definitiva, el conocimiento científico de las cuestiones relacionadas con la sexualidad, permitirían, en el sentir de Hildegart, despojar esta importante faceta de la vida humana de influencias morales represivas y sexistas. Por ese motivo, criticó los métodos pedagógicos tradicionales, ya que, en lugar de ayudar a comprender lo que es la vida sexual, la ocultaban y la convertían en un tabú. Frente a ellos, propuso la instrucción sexual desde la escuela (para lo que sería necesario el apoyo de los poderes públicos) y una educación sexual individualizada partiendo del conocimiento psicológico del niño o del joven.

En este sentido, hay que precisar que la necesidad de una educación sexual de la población dirigida por especialistas era un argumento prácticamente unánime en toda la literatura médica de la época. Los enfoques de la misma divergían, como es lógico, dependiendo de las posiciones ideológicas, políticas y religiosas de cada experto. Así, los médicos más conservadores limitaban sus consejos a la prevención de las enfermedades de transmisión sexual en el caso de los hombres, y a una adecuada preparación de la mujer para el matrimonio y la maternidad. Pero, desde posiciones más progresistas, la educación sexual que debía iniciarse desde la infancia y a la que debían contribuir pedagogos, médicos, psiquiatras y psicoanalistas - era considerada como el elemento nuclear, fundamental e imprescindible en el camino hacia la modernidad sexual.

\section{MEDICINA, PSICOANÁLISIS Y EDUCACIÓN SEXUAL}

En una conferencia sobre "Igualdad sexual" pronunciada en 1927, César Juarros se lamentaba de la habitual "despreocupación por educar al niño en sus primeros años, dejándolo para cuando su psicología está endurecida", y llegaba a afirmar categóricamente: "El niño tiene sexualidad"2. Feliz descubrimiento, sin duda, pero, al mismo tiempo, grave problema, pues eran demasiados años de silencio, represión, ocultación y "educación para la castidad" (Seoane, 2006, 50). En esta tesitura, el médico aparecía como uno de los expertos más capacitados para ofrecer una educación sexual "sobre bases científicas" estrechamente relacionada con la higiene física y mental. En 1930, la Sociedad Española de Higiene premió dos monografías sobre esta misma problemática: la de José Bugallo Sánchez, La Higiene sexual en las escuelas (1939)³, y la de Luis Huerta, La educación sexual del niño y del 
adolescente (1930). Asimismo, la Liga Española de Higiene Mental propuso, entre los temas a tratar en su Congreso de 1932, el de la educación sexual, "por ser esta una cuestión de gran importancia y que interesa a médicos, pedagogos, filósofos, juristas y psiquiatras" (Rodríguez Lafora y Comas, 1947, 9)4.

"¡Hablemos de sexo!"; esta parecía ser pues la consigna generalizada entre el amplio colectivo de profesionales que pensaba que la reforma sexual debía formar parte ineludible de la regeneración social. ¡Hablemos de sexo!, pero hablemos con propiedad, aclarando científicamente las ideas erróneas, las interpretaciones "peligrosas" y, en definitiva, los mitos y las creencias injustificadas, con lo que la "sexuelle Aufklärung" de los alemanes irrumpía con fuerza en la literatura médica española. Esta "revelación de lo sexual" o "ilustración aclaratoria de lo sexual" se arrogaba así la misión de criticar y proscribir las explicaciones mundanas y groseras de la sexualidad, reemplazándolas por informaciones bien medidas que debían favorecer en el niño una sexualidad "honesta" y sin riesgos para la salud o la moral. En palabras del psiquiatra Gonzalo Rodríguez Lafora: "Vale más una ilustración sexual bien dirigida por persona ilustrada que no la ilustración libre adquirida en la calle ("wilde Aufklärung" o aclaración libre de los alemanes)" (Ro-

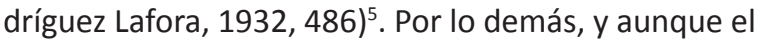
objeto último de la educación sexual era el niño o el adolescente, el discurso pedagógico iba dirigido también, y fundamentalmente, a los padres y a los maestros, ya que, según afirmaba Juarros, "la sexualidad infantil, [está] lamentablemente desatendida por la mayoría o la totalidad de los padres, que no conciben en el niño apetencias sexuales, siendo así que las más arraigadas aberraciones de los sexos tienen su incitación en la misma cuna" ${ }^{6}$.

No podemos dejar de señalar aquí el importante papel que el psicoanálisis llegó a desempeñar en este sentido. El mismo Juarros insistió repetidamente en la importancia del psicoanálisis en la comprensión de la sexualidad infantil, mientras su utilización le parecía necesaria en cualquier programa de educación sexual (Juarros, 1929, 118) 7 . Esta idea fue recogida en un informe presentado por la Liga Española de Higiene Mental al Ministerio de Gobernación en 1933 en el que se afirmaba que "según algunos psicoanalistas, debe incluso tratarse con el niño y el adolescente de todo lo referente a las tendencias incestuosas de ese periodo evolutivo sexual" ${ }^{8}$. Con todo, la aportación más relevante a la discusión sobre la educación sexual desde la órbita del psicoanálisis realizada en la España de los años treinta fue la llevada a cabo por Ángel Garma9 .
En sus publicaciones, Garma discute el concepto de "aclaración de la sexualidad" ("sexuelle Aufklärung"), apuntando que esta debe ser algo más que una "explicación intelectual", ya que es necesario "exponer al niño no solamente cómo es la sexualidad del adulto, sino también cómo es la sexualidad infantil y cómo debe manejar sus tendencias sexuales infantiles" (Garma, 1936, 118-119) ${ }^{10}$. Para este autor, la educación sexual debe comenzar, pues, desde el primer momento en que el niño demanda algún tipo de explicación: "Como el interés del niño va paso a paso y de un terreno a otro, la explicación debe seguir constantemente el mismo camino del interés y debe ir a la misma velocidad" (Garma, 1936, 117-118). En su opinión, la aclaración hecha en la pubertad o en época posterior apenas tiene valor e, incluso, puede ser contraproducente, sobre todo si, hasta ese momento, la educación ha estado ligada a la represión sexual. De hecho, el adolescente educado para rechazar o negar la sexualidad podría desarrollar una resistencia frente a esta ilustración tardía, lo que le llevaría a avivar sus represiones y a desconfiar de las personas de su alrededor.

Para Garma, veracidad y tolerancia son, en definitiva, los dos elementos fundamentales que el adulto responsable debe tener en cuenta al abordar la sexualidad infantil desde un punto de vista médicopedagógico. Los ejemplos que utiliza para ilustrar esta recomendación son suficientemente expresivos. En relación a la veracidad, afirma que "el niño al que se le obliga a creer que los niños vienen de París, se le estropea en parte la lógica del pensamiento" (Garma, 1936, 118) ${ }^{11}$.

En cuanto a la tolerancia, la actitud del adulto padres o educadores - ante la masturbación es, obviamente, una de las mejores reflexiones a las que se puede recurrir. Para Garma, "el niño satisface sus deseos genitales por medio de la masturbación" (Garma, $1936,43)^{12}$. Tal afirmación, planteada así, con toda naturalidad, rompe con las creencias, arrastradas desde siglos, de que el onanismo es perjudicial para la salud física, mental y moral del individuo (Seoane, 2004). Es más, la masturbación es vista como la posibilidad más lógica que tiene el niño (y la niña) para "descargar" su libido (energía sexual). El consejo de Garma es, en consecuencia, la tolerancia, esto es, no interferir en lo que forma parte de la evolución y el desarrollo normal de la sexualidad infantil:

"Si se prohíbe [al niño] la masturbación amenazándole con toda clase de castigos, el niño seguirá mas- 
turbándose secretamente; pero esta masturbación irá acompañada de sentimientos de culpabilidad y por tanto, de remordimientos y de neurosis" (Garma, $1936,118)$.

Cabe precisar, en todo caso, que tal afirmación, hecha en 1934 y aparentemente asumida ya por un número importante de psiquiatras y psicoanalistas, no fue compartida sin reservas por otros autores de reconocido prestigio que, incluso comprometidos con una reforma social de corte más o menos progresista, seguían siendo moderadamente conservadores en aspectos relacionados con la sexualidad (Rodríguez Lafora, 1932, 548 y 549).

Como puede verse, el objetivo de Garma en relación con la educación sexual no estaba orientado, al menos de una manera explícita, ni hacia una moral de la conducta ni hacia la reforma sexual; sus propuestas se encaminaban a conseguir que el niño y el joven sublimaran el curso de la libido, evitando la represión y el sentimiento de culpa - cuya consecuencia sería la neurosis - e impidiendo que dicha energía sexual quedara estancada en alguna fase evolutiva anterior (oral, anal-sádica o genital) y fuera causa de perversiones en el adulto. Su objetivo, en definitiva, no era otro que la prevención de los trastornos psíquicos que, supuestamente, tenían su origen en una sexualidad infantil reprimida. Pero, aunque fuera sin proponérselo - dado que el enfoque de Garma era eminentemente clínico y preventivo-, no cabe duda de que sus planteamientos psicoanalíticos apuntaban claramente a la modernidad sexual, favoreciendo un cambio de costumbres y creencias en torno a la sexualidad. Como ahora mismo veremos, en otras ocasiones estos planteamientos fueron formulados de un modo mucho más explícito y tuvieron consecuencias políticas y sociales de gran alcance, inspirando directamente medidas como el Proyecto de Ley de Divorcio que sería finalmente aprobado por la Cortes en 1932.

\section{REFORMA SEXUAL Y LEGISLACIÓN}

El 2 de marzo de 1932 se promulgó la primera Ley de Divorcio de la historia de España. Se trata, sin duda, de la realización más importante en materia de legislación matrimonial de la Segunda República española. Como era de esperar, en el debate del proyecto de Ley se alzaron voces conservadoras en contra, pero, finalmente, la ley fue aprobada. Sus resultados rebasaron con creces los aspectos meramente normativos, pues, además de las consecuencias sociales y culturales de una nueva concepción del matrimonio y de la familia o, en el plano político, del afianzamiento de un Esta- do laico, la Ley vino también a sancionar, al menos en un cierto sentido, la llamada reforma sexual. De todos modos, la Ley no hacía sino responder a un mandato constitucional, pues el artículo 43 de la Constitución republicana del año anterior ya indicaba que "el matrimonio se funda en la igualdad de derechos para ambos sexos, y podrá disolverse por mutuo disenso o a petición de cualquiera de los cónyuges, con alegación en este caso de justa causa".

No insistiremos aquí en los presupuestos ideológicos ni en la significación política de la Ley (Daza, 1992), pero sí en los argumentos médicos y psicoanalíticos que se utilizaron en su defensa. Presentado ante el Congreso de los Diputados el 4 de diciembre de 1931, el Proyecto de Ley fue elaborado por el jurista Luís Jiménez de Asúa, que fue asesorado en materia psicológica por el médico José Sanchis Banús. Ambos eran diputados socialistas, pero también prestigiosos profesionales interesados en la incorporación del psicoanálisis a la criminología y la psiquiatría. De hecho, ya en 1924 Sanchis Banús había relacionado determinados trastornos mentales con la insatisfacción sexual: "En el 70 por ciento de los enfermos psiconeurósicos que he asistido he podido adquirir la convicción firme de que existía un profundo divorcio entre lo que deseaban y lo que habían logrado en materia sexual" (Sanchis Banús, 1924). Por lo demás, Sanchis Banús también tenía cierta experiencia en la valoración de problemas sexuales en el matrimonio, ya que años antes había actuado como perito en un pleito canónico de nulidad matrimonial en el que declaró que la práctica del coitus interruptus impuesta por el marido había ocasionado en la mujer severos problemas neuróticos directamente derivados de la ansiedad producida por una libido insatisfecha y reprimida (Sanchis Banús, 1923).

En este sentido, es interesante señalar cómo este afán de higiene mental y el interés por prevenir los trastornos mentales relacionados con la represión o la insatisfacción sexual pasó de lo individual a lo colectivo. De hecho, los argumentos psicoanalíticos utilizados en defensa del proyecto de Ley de Divorcio en el parlamento español fueron contundentes a este respecto. El divorcio venía a ser así una forma de prevención de la histeria, al proporcionar a la mujer un medio legal de protegerse y de evitar una "reacción por falta de defensa". Ante la indefensión de la mujer en el seno de la vida conyugal, ante las imposiciones del marido, ante la "conspiración social contra los derechos de la mujer", el divorcio aparecía, pues, como una vía de liberación personal y de profilaxis mental: 
“¿Cómo podemos extrañarnos de que la mujer tenga reacciones en el sentido histérico, si nosotros le cerramos el camino normal de la reacción? Yo sostengo, no que entronizamos el histerismo, sino que anulamos el histerismo por causas de matrimonio, cuando damos a la mujer un medio legal de destruir el vínculo conyugal" 13

La Ley de Divorcio, en suma, constituye un ejemplo muy significativo de cómo la psicología freudiana, la reforma sexual y la defensa de los derechos de la mujer se aunaron ante el problema de la disfunción conyugal. No ocurrió lo mismo con otros aspectos relevantes de la problemática sexual, y, de hecho, el propio Jiménez de Asúa descartó entonces la posibilidad de presentar un proyecto de despenalización del aborto.

Como es sabido, a lo largo de las primeras décadas del siglo XX, pero sobre todo durante los años veinte y treinta, tuvo lugar en España un complejo proceso de modernización social y cultural que afectó a todos los órdenes de la vida, y que llegó a su punto culminante durante la Segunda República ${ }^{14}$. En el ámbito específico que nos ocupa, nos parece incuestionable que tanto la nueva ciencia sexológica como las contribuciones de la psiquiatría y el psicoanálisis aportaron elementos discursivos y propiciaron iniciativas políticas tendentes a una profunda reforma de las costumbres sexuales en una sociedad cada vez más urbana, laica y democrática. Un camino hacia la modernidad sexual que, como hemos apuntado, se truncó tras la Guerra Civil, cuando la Dictadura franquista y el nacionalcatolicismo suprimieron durante años las libertades políticas, pero también impusieron una "moral" que impregnó de manera opresiva y represiva la cultura y la vida cotidiana y, por supuesto, la sexualidad de los españoles (Martínez y Adam, 2004; Regueillet, 2011; Medina, 2013).

\section{AGRADECIMIENTOS}

El presente trabajo ha sido realizado en el marco del Proyecto de investigación HAR2012-37754-C02-01 (Ministerio de Ecomonía y Competitividad).

\section{NOTES}

1 El caso Hildegart conmocionó a la opinión pública, cuando esta niña prodigio - militante del Partido Socialista primero y del Partido Republicano Federa más tarde, feminista, especializada en sexología y competente interlocutora de científicos y políticos-, fue asesinada por su propia madre, Aurora Rodríguez. Una madre convertida en guardiana y protectora de su hija, de la que no se separaba nunca y a la que presentaba siempre como una creación suya, y que, ante el peligro de perderla $-y$ de que finalmente se emancipara de su tutela - acabó por quitarle la vida el 9 de junio de 1933, cuando Hildegart tan solo contaba dieciocho años de edad. La historia de ambas mujeres, así como el análisis del caso criminal y del debate sobre el diagnóstico psiquiátrico de Aurora Rodríguez han sido objeto de diversos estudios. Véanse, por ejemplo, Álvarez y Huertas (1987), Rendueles (1989) o Huertas (2008).

2 El Sol, 27 de noviembre de 1927. La conferencia aludida fue pronunciada por Juarros en la sede de la Federación Tabaquera (Centro de Cigarreras y Tabaqueras), el más importante sindicato femenino de la época y uno de los ejes de las actividades feministas. Véase Glick (1981). César Juarros, prestigioso médico que llegó a ser muy conocido por su actividad publicista y divulgadora llegó a ser, durante el primer bienio republicano, diputado por Derecha Liberal Republicana, incorporándose tras la desaparición de dicha fuerza política al partido Republicano Progresista.

3 En este texto y en otros de la misma época ya están presentes las ideas psicoanalíticas sobre la sexualidad infantil. Otro buen ejemplo puede ser Escalante (1933).

4 El libro de Gonzalo R. Lafora y Margarita Comas, publicado en la República Argentina en 1947, recoge dos textos, uno de Lafora: "La educación sexual", y otro de Margarita Comas: "La coeducación de los sexos", que habían aparecido con anterioridad en la Revista de Pedagogía. La cita está tomada del "Prefacio" de Lafora para el texto de esta última edición. En lo sucesivo se citará por el original de 1932.

5 Sobre la aportación de Lafora al discurso sexual desarrollado en España a prin- cipios del siglo XX puede verse Nistal (2007).

6 La cita textual corresponde a una conferencia pronunciada por Juarros en el Ateneo de Divulgación Social en 1929 y titulada "El placer ajeno". La información sobre la misma fue cubierta por $E I$ Sol, 19 de febrero de 1929. Véase Glick $(1981,18)$.

7 Sobre el peculiar manejo que este autor hace del psicoanálisis, véase Samblás (2008).

8 Este texto, al parecer, fue elaborado por Emilio Mira y López. Véase Glick (1981, 18).

9 Sobre este autor, pueden verse, entre otros, Huertas (2002, 61 y ss.) y Markez (2005).

10 La cita corresponde a un artículo publicado originariamente por Garma (1934) en la Revista de Escuelas Normales. El trabajo se reprodujo dos años más tarde, con el título "Psicoanálisis y pedagogía. Valor psicológico de la aclaración de la sexualidad", como capítulo en la monografía titulada El psicoanálisis, la neurosis y la sociedad. Se cita por esta edición. 
11 Otros autores se muestran más cautos; Lafora, por ejemplo, está convencido de que "este sentimiento de lo natural como primera fase de la ilustración sexual tiene sus límites, pues una excesiva verdad puede perjudicar al niño" (Rodríguez Lafora, 1932, p. 484).
12 La cita corresponde a un artículo publicado originariamente por Garma (1932) en Archivos de Neurobiología. El trabajo se reprodujo en 1936 como un capítulo (pp. 42-50) de la monografía titulada $E l$ psicoanálisis, la neurosis y la sociedad. Se cita por esta edición.

de la homosexualidad masculina en la España del siglo XX". En Martínez-Pérez, J.; Porras, M. I.; Samblás, P.; del Cura, M. (coords.), La medicina ante el nuevo milenio: una perspectiva histórica. Cuenca: Universidad de Castilla-La Mancha, pp. 895-919.

Cleminson, R. (2004). "El libro Homosexualidad del Dr. Martín de Lucenay: entre el conocimiento científico y la recepción pública de la ciencia sexológica en España principios del siglo XX". Hispania, 64 (3), 961-986.

Daza, J. (1992). “La ley de divorcio de 1932. Presupuestos ideológicos y significación política". Alternativas. Cuadernos de Trabajo Social, 1, 163-175.

Ellis, H. (1913). Estudios sobre psicología sexual. Hombre y mujer. Madrid: Hijos de Reus editores.

Ellis, H. (1933). "The red virgin". The Adelphi, June, pp. 174-179.

Balaguer, E. (2013). “Marañón y la medicina en España". Arbor, 189 (759): a002. doi: http://dx.doi.org/10.3989/ arbor.2013.759n1001.

Barrachina, M. A. (2004). "Maternidad, feminidad, sexualidad. Algunos aspectos de las Primeras Jornadas Eugénicas Españolas (Madrid, 1928 - Madrid, 1933)". Hispania, 64 (3), 1003-1026.

Bugallo Sánchez, J. (1930). La Higiene sexual en las escuelas. Madrid: Javier Morata editor.

Castejón, R. (2013). “Marañón y la identidad sexual: biología, sexualidad y género en la España de la década de 1920". Arbor, 189 (759): a005. doi: http://dx.doi. org/10.3989/arbor.2013.759n1004.

Cleminson, R. (1995). Anarquismo y homosexualidad. Antología de artículos de La Revista Blanca, Generación Consciente. Estudios e Iniciales (1924-1935). Madrid: Huerga y Fierro editores.

Cleminson, R. (2004). "Conocimiento marginal/centralidad discursiva: la aberración, la morbosidad y el exceso sexual como dispositivos en la construcción
13 La intervención de Sanchis Banús está recogida en el Diario de Sesiones de las Cortes Constituyentes de la II Repúbli$c a, \mathrm{n}$ 57, 15 de octubre de 1931, pp. 1759-1764.

14 Véase, en este sentido, el reciente ensayo de Juliá (2010).

Hildegart (1931). Paternidad voluntaria: profilaxis anticoncepcional. Valencia: Orto.

Hildegart [1931a] (1971). El problema sexual tratado por una mujer española. Madrid: Morata.

Hildegart (1931b). Educación sexual. Madrid: Gráfica socialista.

Hildegart [1931c] (1977). La rebeldía sexual de la juventud. Prólogo de Eduardo de Guzmán. Barcelona: Anagrama.

Hildegart (1932). Malthusianismo y neomalthusianismo. El control de la natalidad. Madrid: Morata.

Huerta, L. (1930). La educación sexual del niño y del adolescente. Madrid: Instituto Samper.

Huertas, R. (2002). Los médicos de la mente. De la neurología al psicoanálisis. Madrid: Nívola.

Huertas, R. (2008). "Las heridas de la ciencia. A propósito del caso Hildegart". En Fernández, P. y Ortega, M. L. (eds.), La mujer de letras o la letra herida. Discursos y representaciones sobre la mujer escritora en el siglo XIX. Madrid: CSIC, pp. 243-260.

Juarros, C. (1929). Los horizontes del psicoanálisis. Madrid: Mundo Latino.

Juarros, C. (s.a.). Normas de Educación Sexual y Física. Madrid: Renacimiento.

Juliá, S. (2010). Hoy no es ayer. Ensayos sobre la España del siglo XX. Barcelona: RBA.

Marañón, G. (1915). La doctrina de las secreciones internas. Madrid: Biblioteca Corona.

Marañón, G. (1929). Los estados intersexuales en la especia humana. Madrid: Javier Morata.

Markez, I. (2005). El bilbaíno Ángel Garma (1904-1993) fundador del psicoanálisis argentino. Bilbao: BBK.

Martí Ibáñez, F. (1976). Consultorio Psíquico-sexual (De la revista Estudios), 19361937. Barcelona: Tusquets. 
Martínez, A. y Adam, A. (2004). "Consideraciones sobre tan repugnante tendencia sexual: La homosexualidad en la psiquiatría del franquismo". Orientaciones: revista de homosexualidades, 7, 51-81.

Medina, R. (2013). Ciencia y sabiduría del amor. Una historia cultural del franquismo (1940-1960). Frankfurt-Madrid: Vervuert-Iberoamericana.

Nash, M. (1995). "La reforma sexual en el anarquismo español”. En Hofmann, B.; Tous, P. y Tietz, M. (eds.), El anarquismo español y sus tradiciones culturales. Frankfurt-Madrid: Vervuert-lberoamericana, pp. 281-296.

Nistal, M. D. (2007). "Lafora: Sexólogo y Reformador". En Campos, R.; Villasante, O. y Huertas, R. (eds.), De la "Edad de Plata" al exilio. Construcción y "reconstrucción" de la Psiquiatría española. Madrid: Frenia, pp. 59-71.

Noguera, E. (1934). "Cómo se yuguló la generosa idea del Primer Curso Eugénico Español". En Noguera, E.; Huerta, L. (dir.), Genética, eugenesia y pedagogía sexual. Primeras Jornadas Eugénicas Españolas. 2 tomos. Madrid: Morata, t. II, pp. 405-406.

Piga, A. (1910). Higiene de la Pubertad. Toledo: Vda e Hijos de J. Peláez.

Reguéillet, A. G. (2011). "Norma sexual y comportamiento cotidianos en los diez primeros años del franquismo: noviazgo y sexualidad". En Guereña, J. L. (ed.), La sexualidad en la España contemporánea (1800-1950). Cádiz: Universidad de Cádiz, pp. 229-245.
Reich, W. (1932). Der sexuelle Kampf der Jugend. Berlin-Wien-Leipzig: Verlag für Sexualpolitik.

Rendueles, G. (1989). El manuscrito encontrado en Ciempozuelos: Análisis de la historia clínica de Aurora Rodríguez. Madrid: Endymión.

Rodríguez Lafora, G. (1932). “La educación sexual". Revista de Pedagogía, 11, 481490 y 543-549.

Rodríguez Lafora, G. y Comas, M. (1947). La educación sexual y la coeducación de los sexos. Buenos Aires: Losada.

Rodríguez Ocaña, E. (1990). “La asistencia colectiva en España, hasta 1936". En Álvarez Junco, J. (coord.), Historia de la acción social pública en España. Beneficencia y previsión. Madrid: Ministerio de Trabajo y Seguridad Social, pp. 321-359.

Samblás, P. (2008). "El doctor César Juarros (1879-1942) y el diván: 'la psicoanálisis del repentismo'". En Martínez-Pérez, J.; Estévez, J.; del Cura, M. y Blas, L. (eds.), La gestión de la locura. Conocimiento, prácticas y escenarios (España, siglos $X I X-X X)$. Cuenca: Universidad de CastiIla-La Mancha, pp. 507-526.

Sánchez Granjel, L. (1974). “El sexo como problema en la España contemporánea (pesquisa bibliográfica)". Cuadernos de Historia de la Medicina, 13: 111-131.

Sanchis Banús, J. (1923). "Acerca de los trastornos nerviosos originados en la mujer por la práctica sistemática del coitus interruptus y su patogenia". Los Progresos de la Clínica, 26, 196-230.
Sanchis Banús, J. (1924). "La cuestión del psicoanálisis". Archivos de Medicina, Cirugía y Especialidades, 15, 136-142.

Santonja, G. (1989). La República de los libros. El nuevo libro popular en la II República. Barcelona: Anthropos.

Seoane, J. B. (2006). El placer y la norma. Genealogía de la educación sexual en la España contemporánea. Barcelona: Octaedro.

Seoane, J. B. (2004). "Onanismo y perversión: el discurso de los saberes 'expertos' en la España de finales del siglo XIX y principios del siglo XX". En MartínezPérez, J.; Porras, M. I.; Samblas, P.; del Cura, M. (coords.), La medicina ante el nuevo milenio: una perspectiva histórica. Cuenca: Ediciones de la Universidad de Castilla-La Mancha, pp. 879-894.

Sinclair, A. (2003). "The World League for Sexual Reform in Spain: Founding, Infighting, and the Role of Hildegart Rodríguez". Journal of the History of Sexuality, 12 (1), 98-109.

Vázquez, F. y Cleminson, R. (2011). Los invisibles. Una historia de la homosexualidad masculina en España. 1850-1939. Granada: Comares.

Vázquez, F.; Moreno Mengíbar, A. (1997). Sexo y razón. Una genealogía de la moral sexual en España (siglos XVI-XX). Madrid: Akal. 